\title{
Gene co-expression networks in whole blood implicate multiple interrelated molecular pathways in obese asthma
}

\author{
Damien C. Croteau-Chonka, Ph.D. ${ }^{1}$, Zhanghua Chen, Ph.D. ${ }^{2}$, Kathleen C. Barnes, Ph.D. ${ }^{3}$, \\ Albino Barraza-Villarreal, Sc.D. ${ }^{4}$, Juan C. Celedón, M.D.,Dr.P.H. ${ }^{5}$, W. James Gauderman, \\ Ph.D. ${ }^{6}$, Frank D. Gilliland, M.D.,Ph.D. ${ }^{2}$, Jerry A. Krishnan, M.D.,Ph.D. ${ }^{7}$, Andrew H. Liu, M.D. ${ }^{8}$, \\ Stephanie J. London, M.D.,M.P.H., Dr.P.H. ${ }^{9}$, Fernando D. Martinez, M.D. ${ }^{10}$, Joshua Millstein, \\ Ph.D. ${ }^{6}$, Edward T. Naureckas, M.D. ${ }^{11}$, Dan L. Nicolae, Ph.D. ${ }^{12,13,14}$, Steven R. White, M.D. ${ }^{11}$, \\ Carole Ober, Ph.D. ${ }^{12}$, Scott T. Weiss, M.D.,M.S. ${ }^{1,15}$, and Benjamin A. Raby, M.D.,M.P.H. ${ }^{1,16}$ \\ on behalf of Asthma BioRepository for Integrative Genomic Exploration (Asthma BRIDGE) \\ Consortium
}

${ }^{1}$ Channing Division of Network Medicine, Department of Medicine, Brigham and Women's Hospital and Harvard Medical School, Boston, MA, USA

${ }^{2}$ Division of Environmental Health, Department of Preventive Medicine, Keck School of Medicine, University of Southern California, Los Angeles, CA, USA

${ }^{3}$ Division of Biomedical Informatics and Personalized Medicine, Department of Medicine, University of Colorado School of Medicine, Anschutz Medical Campus, Aurora, CO, USA

${ }^{4}$ National Institute of Public Health, Cuernavaca, Morelos, México

${ }^{5}$ Division of Pulmonary Medicine, Allergy and Immunology, Children's Hospital of Pittsburgh of the University of Pittsburgh Medical Center, University of Pittsburgh, Pittsburgh, PA, USA

${ }^{6}$ Division of Biostatistics, Department of Preventive Medicine, Keck School of Medicine, University of Southern California, Los Angeles, CA, USA

${ }^{7}$ Division of Pulmonary, Critical Care, Sleep, and Allergy, Department of Medicine, University of Illinois at Chicago, Chicago, IL, USA

${ }^{8}$ Division of Allergy and Clinical Immunology, Department of Pediatrics, National Jewish Health and University of Colorado School of Medicine, Denver, CO, USA

${ }^{9}$ Division of Intramural Research, Department of Health and Human Services, National Institute of Environmental Health Sciences, National Institutes of Health, Research Triangle Park, NC, USA

${ }^{10}$ Arizona Respiratory Center and BIO5 Institute, University of Arizona, Tucson, AZ, USA

Corresponding Author: Damien C. Croteau-Chonka, Ph.D.181 Longwood Avenue, Boston, MA 02115-5804, damien.croteauchonka@channing.harvard.edu, Office: 617-525-2015.

Author Contributions

- D.C.C.-C. and B.A.R. designed the study.

-D.C.C.-C. performed the analyses.

• K.C.B., A.B.-V., J.C.C., W.J.G., F.D.G., J.A.K., A.H.L., S.J.L., F.D.M., J.M., E.T.N., D.L.N., S.R.W., C.O., S.T.W., and B.A.R. supervised the primary data generation.

- Z.C. curated additional phenotype data for analysis.

- D.C.C.-C. and B.A.R. wrote the manuscript.

All co-authors read and approved the final manuscript. 
${ }^{11}$ Section of Pulmonary and Critical Care Medicine, Department of Medicine, University of Chicago, Chicago, IL, USA

${ }^{12}$ Department of Human Genetics, University of Chicago, Chicago, IL, USA

${ }^{13}$ Section of Genetic Medicine, Department of Medicine, University of Chicago, Chicago, IL, USA

${ }^{14}$ Department of Statistics, University of Chicago, Chicago, IL, USA

${ }^{15}$ Partners HealthCare Personalized Medicine, Partners Health Care, Boston, MA, USA

${ }^{16}$ BWH Pulmonary Genetics Center, Division of Pulmonary and Critical Care Medicine, Department of Medicine, Brigham and Women's Hospital and Harvard Medical School, Boston, MA, USA

\section{Abstract}

Objective: Asthmatic children who develop obesity through adolescence have poorer disease outcomes compared to those that do not. We aimed to characterize the biology of childhood asthma complicated by adult obesity.

Methods: Gene expression networks are powerful statistical tools for characterizing human disease that leverage the putative co-regulatory relationships of genes to infer relevant biological pathways. We performed weighted gene co-expression network analysis (WGCNA) of gene expression data in whole blood from 514 adult asthmatic subjects. We then performed module preservation and association replication analyses in 418 subjects from two independent asthma cohorts (one pediatric and one adult).

Results: We identified a multivariate model in which three gene co-expression network modules were associated with incident obesity in the discovery cohort (each $P<0.05$ ). Two module memberships were enriched for genes in pathways related to platelets, integrins, extracellular matrix, smooth muscle, NF- $\mathrm{kB}$ signaling, and Hedgehog signaling. The network structures of each of the obese asthma modules were significantly preserved in both replication cohorts (permutation $P=9.999 \mathrm{E}-05$ ). The corresponding module gene sets were significantly enriched for differential expression in subjects who were obese in both replication cohorts (each $P<0.05$ ).

Conclusions: Our gene co-expression network profiles thus implicate multiple interrelated pathways in the biology of an important endotype of obese asthma.

\section{Keywords}

asthma; obesity; gene expression; blood

\section{Introduction}

Obesity is recognized as an important risk factor for, and a major co-morbidity of, asthma in both children and adults $(1,2)$. Obesity is associated with a significantly higher risk of developing asthma, worse asthma symptoms, poor asthma control, and greater resistance to standard asthma therapies (1). A recent review (2) postulates at least three (likely four) endotypes of obese asthma $(3,4)$, distinguished by the relative timings of each disease's onset (onset during childhood versus adulthood) and by their underlying pathophysiologies 
(differing effects of obesity on airway structure, function, sensitivity, and/or inflammation). A more complete understanding of the complex molecular relationships between obesity and asthma would drive the development of specific and effective preventative and therapeutic strategies for this overlap syndrome.

Despite large numbers of published studies characterizing global gene expression of each condition separately, there are few examples of comprehensive blood transcriptional profiling studies assessing the interrelationships of asthma and obesity. Of the three published studies $(5,6,7)$ to date, only one (5) directly compared gene expression profiles between asthmatic individuals with and without obesity; it was of small sample size $(n<10$ per group) and lacked replication. Furthermore, all three studies were cross-sectional, making it difficult to pinpoint which endotypes of obese asthma they were characterizing. With these limitations, the three studies cumulatively implicated pathways related to the adaptive and innate immune systems in obese asthma. Larger, longitudinal studies are needed to determine the generalizability and reproducibility of these findings.

To address these knowledge gaps, we performed weighted gene co-expression network analysis (WGCNA) (8) in a cohort of asthmatic children who were followed longitudinally into young adulthood. Gene co-expression networks are powerful statistical tools for characterizing the underpinnings of human disease that use the putative co-regulatory relationships of genes to infer the biological pathways altered in disease states. We studied children who participated in the Childhood Asthma Management Program (CAMP), a fouryear clinical trial and 12-year natural history follow-up study of the effects of inhaled antiinflammatory medications in patients with mild to moderate persistent asthma (9). A prior analysis in CAMP showed that asthmatic children who became obese during the observation period had impaired growth in lung function compared to those who did not (10). To determine whether these children had a distinct molecular profile as adults that could contribute to this association, we performed WGCNA using gene expression data from peripheral blood collected at early adulthood available from a subset of 514 CAMP participants, including 104 (20\%) that became obese by adulthood (incident obesity). We then replicated our findings in two additional, independent asthma cohorts (one pediatric and one adult, totaling 418 subjects).

Through this effort, we discovered a set of gene co-expression network modules that were reproducibly associated with obesity in asthma. These modules were enriched for genes linked to several important molecular processes, implicating platelet, integrin, extracellular matrix, smooth muscle, NF- $\mathrm{kB}$ signaling, and Hedgehog (Hh) signaling pathways in the biology of obese asthma.

\section{Methods}

The methodological motivations of this study are described in detail in the Supporting Information. We provide here a brief summary. 


\section{Asthma study cohorts}

The CAMP study was initiated as a randomized clinical trial studying the long-term effects of three asthma medications on disease progression (9) (https://clinicaltrials.gov identifier NCT00000575). Subjects in this cohort had mild-to-moderate asthma and were phenotyped longitudinally from childhood (aged five to 12 years at baseline of the trial) until early adulthood, as previously described (10).

Replication was performed in two independently ascertained asthma cohorts: The Asthma BioRepository for Integrative Genomic Exploration (Asthma BRIDGE) and the Genetics of Asthma in Costa Rica Study (GACRS). Asthma BRIDGE consists of an ethnically diverse set of asthmatic adults from the United States who were studied as part of the EVE asthma genetics consortium (11) and who were further characterized with blood gene expression profiles (12). For the current study, we used a subset of adult Asthma BRIDGE subjects from the Southern California Children's Health Study (CHS) for whom both longitudinal asthma and obesity phenotypes were available, spanning childhood (aged 5 to 8 years of age at study enrollment) until early adulthood (13). GACRS consists of asthmatic children (aged six to 14 years) ascertained from the Central Valley of Costa Rica, a population isolate with a high prevalence of asthma (14). Subjects in GACRS had only cross-sectional data on asthma and obesity phenotypes.

This analysis of human subjects data was approved by the Institutional Review Board of Brigham and Women's Hospital.

\section{Gene expression data}

The cross-sectional CAMP gene expression data have been previously described (12). Briefly, 620 CAMP subjects with whole blood samples collected only at early adulthood were assayed with the Illumina HT-12 (version 3) platform with 47,009 high-quality probes. Quality control filters included removal of failed arrays, probes with low outlying $\log _{2}$ intensities $(<5)$, and probes with poor signal-to-noise ratios $\left(95^{\text {th }}\right.$ percentile $/ 5^{\text {th }}$ percentile). These expression data were quantile-normalized and $\log _{2}$-transformed. A set of 106 subjects who were with obesity at enrollment in CAMP (body mass index (BMI) $\geq 95^{\text {th }}$ percentile for age and sex) were excluded to focus the analysis on asthma complicated by incident obesity (10). Following the exclusion of probes with low variances (15), the final dataset comprised 514 subjects and 10,448 probes. For replication, cross-sectional whole blood gene expression data were also assayed with the Illumina HT-12 array (version 4): 47,009 highquality probes for 91 adult subjects in CHS (12); and 47,256 high-quality probes for 329 pediatric subjects in GACRS (16). A subset of 10,448 probes in CHS and 10,437 probes in GACRS were matched to the CAMP data by their universal nucleotide identifiers (17).

\section{Statistical analyses}

Statistical analyses were performed with Bioconductor in $\mathrm{R}$ (both version 3.3). We performed WGCNA on each gene expression dataset with the R package "WGCNA" (8) (version 1.51). We first characterized unsigned correlation networks and their relationships with each other (18) in the discovery cohort (CAMP) and later examined the properties of those same networks in the replication cohorts (CHS and GACRS). We next tested the 
association of all network modules with the outcome of adult obesity case status using a multivariate linear model. We used the "glmulti" package (version 1.0) to perform automated model selection based on a genetic algorithm (19). Models were ranked based on Bayes Information Criterion (BIC). The parsimonious association model was further adjusted for possible demographic and technical confounders: age, sex, self-reported race/ ethnicity, and measured blood cell counts (\% of eosinophils, monocytes, neutrophils, and lymphocytes). Blood cell counts were imputed for CHS and GACRS based on gene expression using the R package "CellMix" (version 1.6) (20). Eosinophil counts could not be imputed with the default CellMix reference panel, but were directly measured in both replication cohorts.

To assign biological interpretability to the associated modules from the discovery cohort, we performed pathway overrepresentation analyses using g:Profiler (21) (database version: r1730_e88_eg35) and the corresponding R package "gProfiler" (version 0.6). Human Genome Organisation (HUGO) gene symbols served as gene identifiers for each expression probe. We used as the statistical background for the enrichment tests only the set of genes assayed in our data. To increase the specificity of the enrichment results, we set a more stringent threshold of overlap (intersection of query and test sets $\geq 3$ ) and excluded consideration of inferred electronic annotations (i.e., annotations created by in silico curation methods of potentially lower quality). An adjusted $P$ value was calculated in a manner that accounted for the hierarchical relationships among the tested gene sets (21).

Replication analysis in CHS and GACRS consisted of two steps: module preservation and replication of differential gene expression. We tested network module preservation between the discovery and replication cohorts using NetRep (version 1.0) (22). For each replication cohort, 10,000 network permutations were used to determine preservation significance. Replication differential expression analysis and gene set enrichment analysis (GSEA) (23) was performed using the R/Bioconductor package "phenoTest" (version 1.18). This two-step replication analysis served to complement the module association approach used for the discovery cohort. The differential expression model included the same demographic and technical covariates as the network association model tested in the discovery cohort. Gene set enrichment was assessed using a Wilcoxon test (23), which addressed whether the average value of the genes that belonged to a given set was different from the average value of the remaining genes that did not belong to that set. We chose the memberships of the coexpression modules as specific gene sets we hypothesized to be differentially expressed in subjects with obesity.

\section{Results}

\section{Gene co-expression network modules of obese asthma in discovery cohort}

Our study was designed to help better understand the biological processes underlying the relationship of obesity with asthma (Figure 1). For this, we used gene expression profiling of the peripheral blood of subject with childhood asthma from the CAMP cohort, comparing those who did $(n=104)$ and did not $(n=410)$ become obese after approximately 16 years of observation. Obesity during adulthood was defined as a body mass index (BMI) $\geq 30$. All subjects were without obesity during childhood (BMI $<95^{\text {th }}$ percentile). Baseline 
characteristics for the 514 subjects with both complete phenotype and expression data are summarized in Table 1 and in the Supporting Information.

Given the more adverse features of asthma observed in CAMP subjects with obesity versus those without (1), we hypothesized that asthmatic subjects who became obese by adulthood would demonstrate coordinated patterns of global expression that were different from those observed in subjects who remained lean. Furthermore, these observed differences would implicate specific biological pathways that underlie differences between obese and nonobese asthma. To test this hypothesis, we first performed WGCNA on the adult whole blood expression data available in CAMP (Figure 1) (8).

Of the 10,448 genes considered in our analysis, 7,778 clustered into 17 distinct network modules of co-expressed genes (Table S1, Table S2, and Figure S2), which were each assigned an arbitrary color name. The remaining 2,670 genes that could not be assigned membership to a module because of insufficient co-expression were grouped as a collection termed the "null" module. Each module was represented by a summary eigengene (i.e., the first and largest principal component (PC) of variation among the co-expressed genes comprising that module) (Figure 2).

With 17 modules defined, we next identified ones related to obese asthma by testing for differences in each module's eigengene distribution between asthmatics with and without obesity (Supporting Information). We obtained a parsimonious association model of four eigengenes by performing a systematic parameter selection procedure (Figure S3 and Supporting Information). We then examined whether there was evidence of confounding by demographic differences between CAMP subjects with and without obesity that might affect this parsimonious model. We expanded the unadjusted four-eigengene model to include factors that can be associated with blood expression $(12,24)$ : age, sex, race, childhood BMI, blood cell counts, and recent asthma medication usage. Three of the four eigengenes were still robustly associated with adult obesity in this adjusted epidemiological model of obese asthma (all $P<0.01$ ) (Table 2 and Figure 3).

\section{Biological pathways in network modules of obese asthma}

To assign biological interpretability to the four obese asthma modules, we performed pathway enrichment analyses on their corresponding gene memberships. We found that the memberships of only two of these modules (black, midnightblue) were significantly enriched (adjusted $P<0.05$ ) for genes from biological pathways annotated in the Reactome database (Table 3 ). None of the 48 enriched pathways were shared between these two modules. The grey60 and lightcyan modules had no significantly enriched pathways. Negative control gene sets consisting of 50 or 200 random genes from the null module also had no significantly enriched pathways.

The midnightblue module was enriched for four pathways related to platelet and extracellular matrix biology, and for a smooth muscle contraction pathway. A subset of 20 of the midnightblue module's 66 genes (30\%) were responsible for the enrichments seen, with six of these genes shared the across at least two pathways (Table S3). Three of these genes code integrin subunits (ITGA2B, ITGB3, ITGB5), while the others code a cytoskeletal 
protein ( $V C L)$, an extracellular matrix protein (SPARC), and the Von Willebrand Factor platelet glycoprotein $(V W F)$.

The black module was enriched for 44 pathways, including multiple pathways related to mitochondrial and ribosomal biology. These enrichments were driven by 130 of 464 module genes (28\%), 24 of which were shared across five or more pathways. A subset of 34 of the enriched pathways involved the same 13 proteasome subunits, including four sets related to non-canonical NF- $\kappa B$ signaling and NF- $\kappa B$ activation (two of which included NFKB1 itself) (Table S3). Other enriched pathways of interest include those related to interleukin-1 signaling, cystic fibrosis transmembrane conductance regulator (CFTR) dysfunction in cystic fibrosis, and metabolism of the Gli family of transcription factors and other components that all regulate Hh pathway activity.

\section{Preservation and replication of obese asthma modules}

To assess the reproducibility and generalizability of our results, we replicated our analysis in two independent datasets. One replication cohort consisted of 91 asthmatic adults (CHS) and the other consisted of 327 asthmatic children (GACRS). Baseline characteristics for these 418 replication subjects with both complete phenotype and expression data are summarized in Table S4, Table S5, and in the Supporting Information. Replication analysis in CHS and GACRS consisted of two steps: module preservation and module association. Preservation refers to the consistency of network module structure across datasets. If modules are not preserved across cohorts, then their corresponding eigengenes are not comparable, thus precluding further eigengene-phenotype association testing. The seven preservation statistics are motivated in detail elsewhere $(8,22,25)$.

In the first stage of replication, we found very strong evidence of module preservation in the two replication cohorts. All four modules from CAMP were highly significantly preserved in both CHS and GACRS ( $P=9.999 \mathrm{E}-05$ for each, based on permutation testing, Tables S6 and S7). The seven preservation statistics for each of the modules were very similar in magnitude between and within the two replication cohorts. Visualization of the four modules' properties in the replication cohorts further reinforced the notion of strong preservation (Figure 4). Within each module, we observed four important patterns. First, genes were consistently positively correlated with each other but not with genes in other modules, meaning that a network community was a distinct group. Second, the vast majority of gene-pairs had edge weights (correlations) close to zero, reflecting that only a minority of genes were strongly correlated with more than a few other genes. Third, those same genes with higher edge weights had higher weighted degrees, reflecting their increased network connectivity. In other words, genes that were highly connected ("hub-like") in the discovery cohort also acted as module hubs in the replication cohorts. Fourth, node contributions were broadly directionally consistent, meaning that genes were consistently correlated with the corresponding module summary measure (i.e., the eigengene). Thus, the obese asthma network modules identified in CAMP had reproducible architectures in CHS and GACRS.

In the second stage of replication, we tested these highly preserved modules for evidence of phenotypic association with obesity in the two additional asthma cohorts. Testing was done at two levels: (i) assessing replication of each module's eigengene association with obese 
asthma; and (ii) assessing replication at the gene level by GSEA of differential expression of each module's individual component genes. Minimal evidence for replication was observed at the eigengene level: no significant associations were observed in CHS, and although in GACRS the midnightblue eigengene was significantly associated with BMI $Z$-score, its direction of effect was opposite of that observed in the CAMP discovery cohort (Tables S8 and S9).

In contrast, we found stronger replication evidence when considering individual expression differences for each module's gene components using GSEA. In CAMP, three of the module gene sets (except those in the lightcyan module) were significantly enriched for differential expression between asthmatic subjects with and without obesity $(P<0.05)$ (Table 4$)$, in directions consistent with their modules' original eigengene associations (Table 2). In CHS, the enrichments for three of the four modules were similarly significant in the direction consistent with the CAMP results. In GACRS, the enrichments for all four modules were significant in the direction consistent with the CAMP results. Furthermore, negative control gene sets consisting of 50 or 200 random genes from the null module were generally not significantly enriched in any of the three cohorts $(P>0.2)$. These gene set enrichment results paralleled the fact that in each of the four modules, the node contributions of the corresponding genes were very consistent across the three cohorts (Figure 4). These complementary differential gene expression results further supported the existence of four co-expression modules associated with obese asthma.

Finally, we also examined the relationships of our four obese asthma modules with results from a comprehensive whole blood expression profiling study of obesity whose older adult subjects were not ascertained for asthma. Homuth et al. identified 1,913 genes were associated with increased BMI across 1,977 individuals at a false discovery rate $<0.01$ (26). Of the 613 genes from the four obese asthma modules, $496(81 \%)$ were assayed in the Homuth dataset and 156 (25\%) showed significant associations with increased BMI. The partial overlap of these signals suggests that our analyses highlighted a specific component of obesity-related transcriptional programming that is most prominent in asthmatic individuals.

\section{Discussion}

In this work, we used whole blood gene expression profiling to characterize the biology of a form of obese asthma known as "asthma complicated by obesity" (2) (i.e., cases where asthma preceded the development of obesity). Among 514 subjects who were diagnosed with asthma as children, we identified three co-expression network modules jointly associated with adult obesity. We then demonstrated compelling statistical evidence of replication in a total of 418 subjects from two independent asthma cohorts (one adult and one pediatric).

Pre-existing scientific evidence strongly supports the disease relevance of our obese asthma expression signatures. While a detailed survey is presented in the Supporting Information, we highlight here several notable connections. Two of the obese asthma networks, termed the "black" and "midnightblue" modules, were significantly enriched for genes from several 
biological pathways of interest (e.g., platelets, smooth muscle contraction, integrins, and NF- $\kappa$ B signaling). Platelets have been linked to allergic airway disease through both innate and adaptive immune pathways affecting bronchoconstriction, airway remodeling, and airway inflammation $(27,28)$. Airway smooth muscle function has also been shown to be affected by consumption of a high-fat diet (29), a key exposure in the development of obesity. Integrins are transmembrane proteins that anchor airway smooth muscle to its extracellular matrix that may be important novel therapeutic targets in asthma because of their roles in airway hyper-responsiveness and remodeling (30). Finally, a mouse model of obese asthma has suggested that its pathobiology includes increased NF- $\kappa$ B signaling and oxidative stress in lung tissue (31).

Despite the overall strength of our study findings, there are important epidemiological, genomic, and statistical limitations to consider, which are described in full detail in the Supporting Information. We briefly summarize here the primary limitations of our work. First, it is possible that our findings may not be applicable to other endotypes of obese asthma, such as when the development of obesity precedes the development of asthma rather than the other way around. Additionally, our study did not directly include contrasts with non-asthmatic controls (with and without obesity), so we cannot definitively conclude whether the observed signatures are specific to obese asthma or would also be seen in nonasthmatic individuals with obesity. Second, our focus on patterns of gene expression in whole blood samples is unlikely to capture molecular processes that are specific to other disease-relevant tissues, such as lung and adipose. Third, gene co-expression networks characterize the undirected correlational relationships between genes and are therefore not informative about directed causal relationships. Finally, as our direct replication of the eigengene associations with obesity was inconclusive, we had to rely on a complementary approach of demonstrating consistent evidence of differential expression by disease state across the discovery and replication cohorts to support consistent differential co-expression by disease state.

In conclusion, we have identified three robust gene co-expression network modules of asthma complicated by obesity. Nearly every module-related biological pathway we highlighted has been implicated in both asthma and obesity. Together, these findings suggest a few mechanistic possibilities: (1) that subsets of individuals might be genetically programmed with shared pathways that lead to this obese asthma phenotype; or (2) that a subset of asthmatic individuals has a second set of exposures that begets changes in expression resulting in consequent obesity; or (3) that the subsequent development of obesity itself triggers the observed expression change. We propose these candidate genes and pathways merit further examination in ongoing and future epidemiological studies and clinical trials of obese asthma.

\section{Supplementary Material}

Refer to Web version on PubMed Central for supplementary material. 


\section{Acknowledgements}

We thank the Asthma BRIDGE Consortium study staff for their assistance and contributions, as well as all the study subjects for their participation in the Asthma BRIDGE initiative.

Funding

This work was funded by extramural grants from the National Heart, Lung, and Blood Institute at the U.S. National Institutes of Health (NIH): RC2 HL101543 (America Recovery and Reinvestment Act "Grand Opportunity") (B.A.R.), R01 HL086601 (B.A.R.), and K01 HL127265 (D.C.C.-C.). S.J.L. was supported by the NIH Intramural Research Program at the National Institute of Environmental Health Sciences.

Disclosure

Drs. Barnes, Croteau-Chonka, Liu, Martinez, Raby, and White each reports being externally funded by the NIH as part of conducting this study. Dr. Celedón reports relevant financial activities outside of conducting this study: grants from Merck and GlaxoSmithKline; and non-financial support from Pharmavite. Dr. Liu reports relevant financial activities outside of conducting this study: personal fees from Phadia Thermofisher and Merck Sharp \& Dohme; and other support from GlaxoSmithKline. Dr. Martinez relevant reports financial activities outside of conducting this study: other NIH grant support and grants from Johnson \& Johnson; and personal fees from Copeval and Commense Inc. Dr. Raby relevant reports financial activities outside of conducting this study: personal fees from UpToDate, Inc. Dr. White relevant reports financial activities outside of conducting this study: other NIH grant support.

\section{References}

1. Gibson PG. Obesity and asthma. Ann Am Thorac Soc 2013;10 Suppl: S138-142. [PubMed: 24313764]

2. Dixon AE, Poynter ME. Mechanisms of Asthma in Obesity. Pleiotropic Aspects of Obesity Produce Distinct Asthma Phenotypes. Am J Respir Cell Mol Biol 2016;54: 601-608. [PubMed: 26886277]

3. Scott HA, Wood LG, Gibson PG. What About Neutrophils in Obese Asthma? Am J Respir Cell Mol Biol 2016;55: 462.

4. Dixon AE, Poynter ME. Reply: What About Neutrophils in Obese Asthma? Am J Respir Cell Mol Biol 2016;55: 462-463.

5. Gruchala-Niedoszytko M, Niedoszytko M, Sanjabi B, van der Vlies P, Niedoszytko P, Jassem E, et al. Analysis of the differences in whole-genome expression related to asthma and obesity. Pol Arch Med Wewn 2015;125: 722-730. [PubMed: 26252510]

6. Bjornsdottir US, Holgate ST, Reddy PS, Hill AA, McKee CM, Csimma CI, et al. Pathways activated during human asthma exacerbation as revealed by gene expression patterns in blood. PLoS ONE 2011;6: e21902. [PubMed: 21779351]

7. George BJ, Reif DM, Gallagher JE, Williams-DeVane CR, Heidenfelder BL, Hudgens EE, et al. Data-driven asthma endotypes defined from blood biomarker and gene expression data. PLoS ONE 2015;10: e0117445. [PubMed: 25643280]

8. Langfelder P, Horvath S. WGCNA: an R package for weighted correlation network analysis. BMC Bioinformatics 2008;9: 559. [PubMed: 19114008]

9. Childhood_Asthma_Management_Program_Research_Group. The Childhood Asthma Management Program (CAMP): Design, Rationale, and Methods. Control Clin Trials 1999;20: 91-120. [PubMed: 10027502]

10. Strunk RC, Colvin R, Bacharier LB, Fuhlbrigge A, Forno E, Arbelaez AM, et al. Airway Obstruction Worsens in Young Adults with Asthma Who Become Obese. J Allergy Clin Immunol Pract 2015.

11. Torgerson DG, Ampleford EJ, Chiu GY, Gauderman WJ, Gignoux CR, Graves PE, et al. Metaanalysis of genome-wide association studies of asthma in ethnically diverse North American populations. Nature Genetics 2011;43: 887-892. [PubMed: 21804549]

12. Croteau-Chonka DC, Qiu W, Martinez FD, Strunk RC, Lemanske RF, Jr., Liu AH, et al. Gene Expression Profiling in Blood Provides Reproducible Molecular Insights into Asthma Control. Am J Respir Crit Care Med 2017;195: 179-188. [PubMed: 27494826] 
13. Chen Z, Salam MT, Alderete TL, Habre R, Bastain TM, Berhane K, et al. Effects of Childhood Asthma on the Development of Obesity among School-aged Children. Am J Respir Crit Care Med 2017.

14. Hunninghake GM, Soto-Quiros ME, Avila L, Ly NP, Liang C, Sylvia JS, et al. Sensitization to Ascaris lumbricoides and severity of childhood asthma in Costa Rica. J Allergy Clin Immunol 2007;119: 654-661. [PubMed: 17336615]

15. Bourgon R, Gentleman R, Huber W. Independent filtering increases detection power for highthroughput experiments. Proceedings of the National Academy of Sciences of the United States of America 2010;107: 9546-9551. [PubMed: 20460310]

16. Virkud YV, Kelly RS, Croteau-Chonka DC, Celedon JC, Dahlin A, Avila L, et al. Novel eosinophilic gene expression networks associated with IgE in two distinct asthma populations. Clin Exp Allergy 2018.

17. Du P, Kibbe WA, Lin SM. nuID: a universal naming scheme of oligonucleotides for illumina, affymetrix, and other microarrays. Biol Direct 2007;2: 16. [PubMed: 17540033]

18. Langfelder P, Horvath S. Eigengene networks for studying the relationships between co-expression modules. BMC Syst Biol 2007;1: 54. [PubMed: 18031580]

19. Calcagno V, de Mazancourt C. glmulti: An R Package for Easy Automated Model Selection with (Generalized) Linear Models. 2010 2010;34: 29.

20. Gaujoux R, Seoighe C. CellMix: a comprehensive toolbox for gene expression deconvolution. Bioinformatics 2013;29: 2211-2212. [PubMed: 23825367]

21. Reimand J, Arak T, Adler P, Kolberg L, Reisberg S, Peterson H, et al. g:Profiler-a web server for functional interpretation of gene lists (2016 update). Nucleic Acids Research 2016;44: W83-89. [PubMed: 27098042]

22. Ritchie SC, Watts S, Fearnley LG, Holt KE, Abraham G, Inouye M. A Scalable Permutation Approach Reveals Replication and Preservation Patterns of Network Modules in Large Datasets. Cell Syst 2016;3: 71-82. [PubMed: 27467248]

23. Subramanian A, Tamayo P, Mootha VK, Mukherjee S, Ebert BL, Gillette MA, et al. Gene set enrichment analysis: a knowledge-based approach for interpreting genome-wide expression profiles. Proceedings of the National Academy of Sciences of the United States of America 2005;102: 15545-15550. [PubMed: 16199517]

24. Sharma S, Murphy A, Howrylak J, Himes B, Cho MH, Chu JH, et al. The impact of self-identified race on epidemiologic studies of gene expression. Genetic epidemiology 2011;35: 93-101.

[PubMed: 21254216]

25. Langfelder P, Luo R, Oldham MC, Horvath S. Is my network module preserved and reproducible? PLoS Comput Biol 2011;7: e1001057. [PubMed: 21283776]

26. Homuth G, Wahl S, Muller C, Schurmann C, Mader U, Blankenberg S, et al. Extensive alterations of the whole-blood transcriptome are associated with body mass index: results of an mRNA profiling study involving two large population-based cohorts. BMC Med Genomics 2015;8: 65. [PubMed: 26470795]

27. Idzko M, Pitchford S, Page C. Role of platelets in allergic airway inflammation. J Allergy Clin Immunol 2015;135: 1416-1423. [PubMed: 26051948]

28. Page C, Pitchford S. Platelets and allergic inflammation. Clin Exp Allergy 2014;44: 901-913. [PubMed: 24708345]

29. Wood LG, Garg ML, Gibson PG. A high-fat challenge increases airway inflammation and impairs bronchodilator recovery in asthma. J Allergy Clin Immunol 2011;127: 1133-1140. [PubMed: 21377715]

30. Wright DB, Meurs H, Dekkers BG. Integrins: therapeutic targets in airway hyperresponsiveness and remodelling? Trends Pharmacol Sci 2014;35: 567-574. [PubMed: 25441775]

31. Liu X, Lin R, Zhao B, Guan R, Li T, Jin R. Correlation between oxidative stress and the NFkappaB signaling pathway in the pulmonary tissues of obese asthmatic mice. Mol Med Rep 2016;13: 1127-1134. [PubMed: 26677140] 


\section{Study Importance Questions}

- There potentially exist four endotypes of obese asthma distinguished by the relative timings of each disease's onset and by their underlying pathophysiologies.

- Individuals with asthma complicated by obesity had distinct gene coexpression signatures in whole blood implicating several inflammatory biological pathways. 


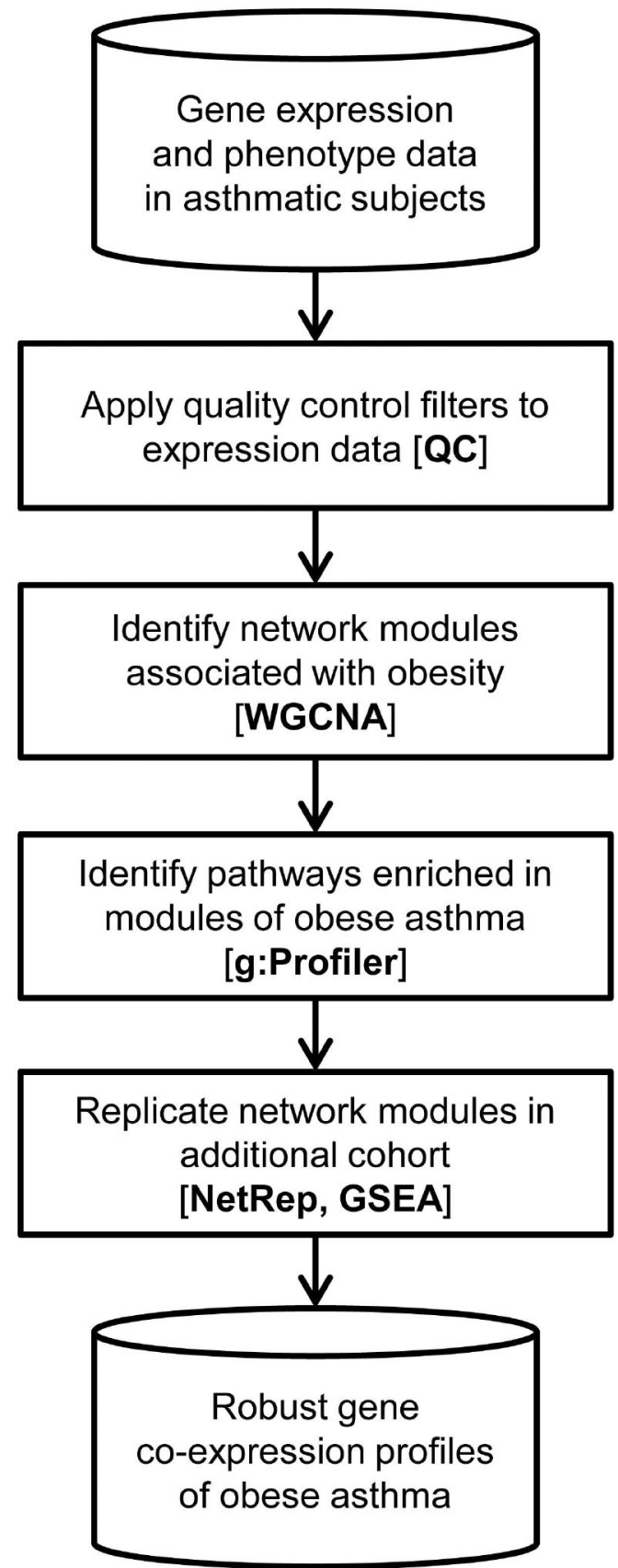

Figure 1: Analysis overview.

In this schematic of this gene expression study of obese asthma, cylinders represent inputs and outputs, and boxes represent analysis steps. More detail for each analysis stage can be found in the Methods section and in the Supporting Information. QC, quality control; WGCNA, weighted gene co-expression network analysis; GSEA, gene set enrichment analysis. 



Figure 2: Hierarchical relationships of 17 modules identified in CAMP.

Top panel shows eigengene dissimilarity clustering. Bottom panel shows pair-wise Pearson correlations among the eigengenes, ranging from -1 (blue) to 1 (red). The order of the colored columns corresponds to the color names in the top panel. Black boxes denote those modules belonging to the larger and smaller meta-modules. Asterisks denote modules associated with obese asthma in multivariate regression modeling (Table 2). ME, module eigengene. 

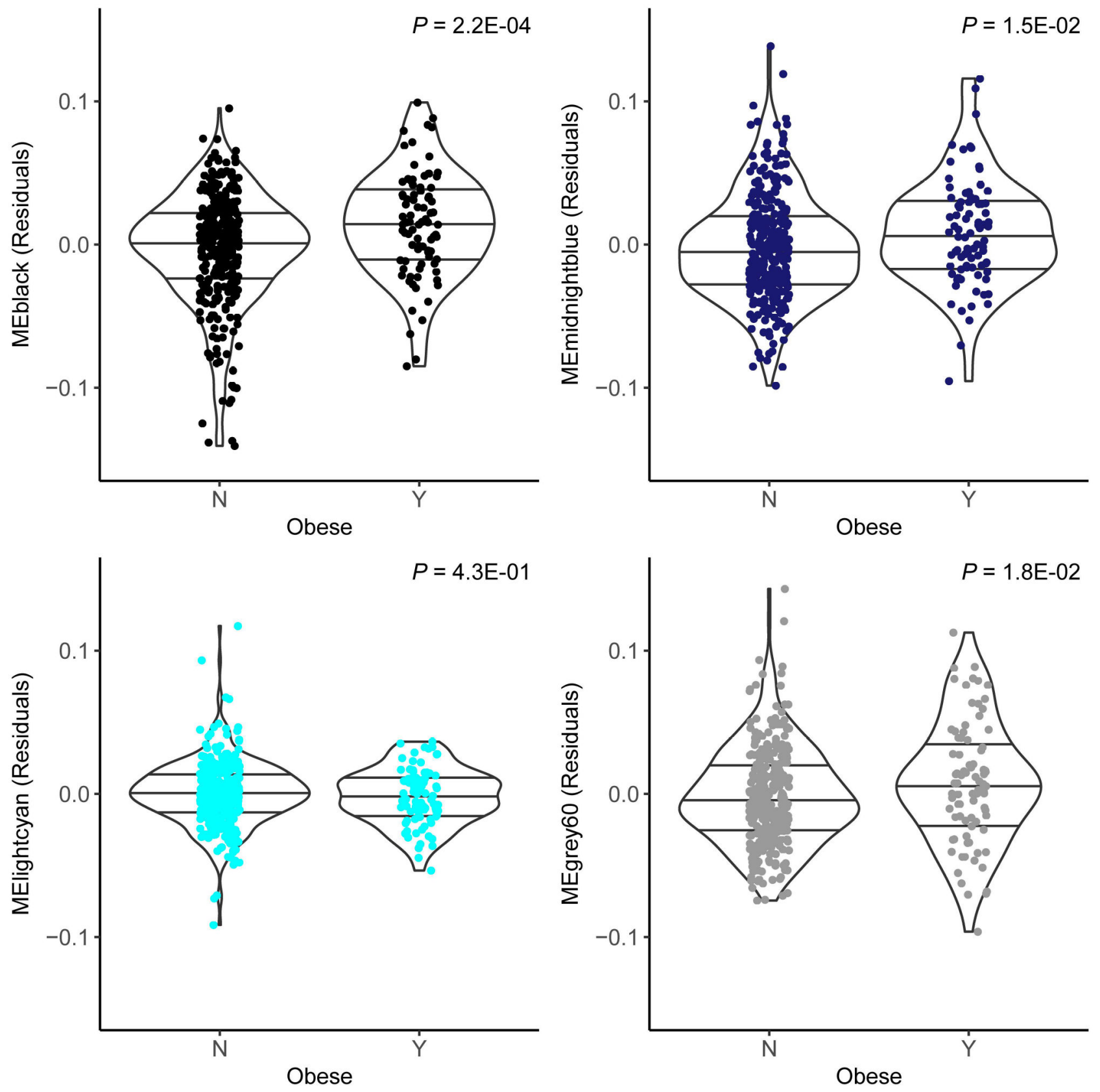

Figure 3: Associations of four eigengenes with obese asthma in CAMP.

Panels show violin plots of eigengene residuals by obesity status. Residuals include adjustments for demographic characteristics and the other three eigengenes (see Table 2). Each dot represents an individual CAMP subject. Horizontal lines indicate $25^{\text {th }}, 50^{\text {th }}$, and $75^{\text {th }}$ percentiles. In this multivariate context, the black, midnightblue, and grey60 eigengenes are significantly different between obese and non-obese asthmatic subjects $(P<0.05)$, while the lightcyan eigengene is not. 


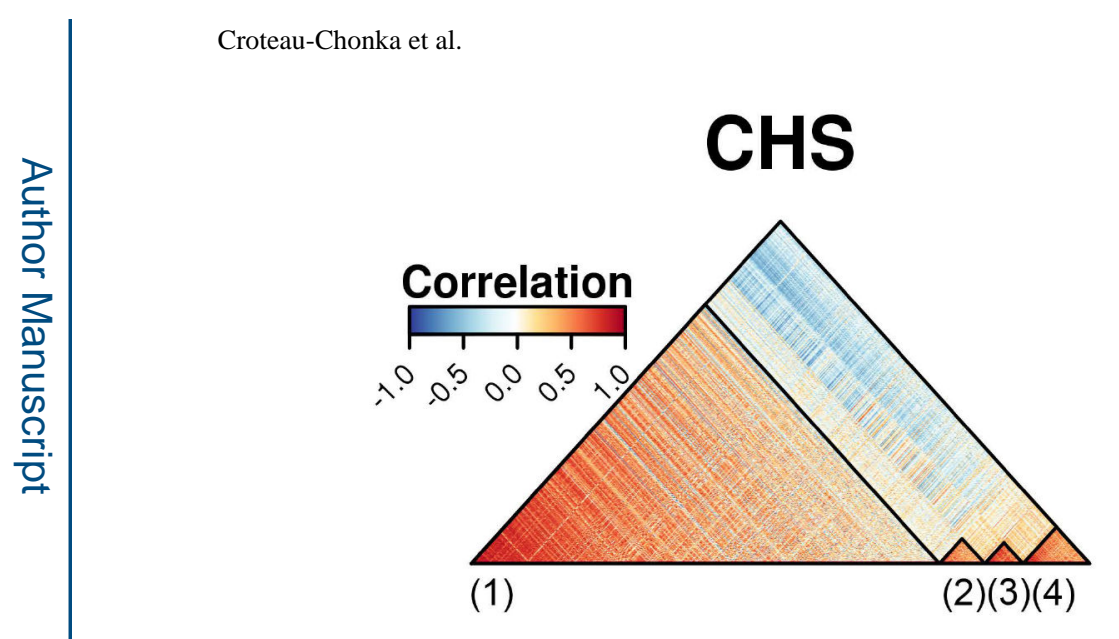

\section{GACRS}
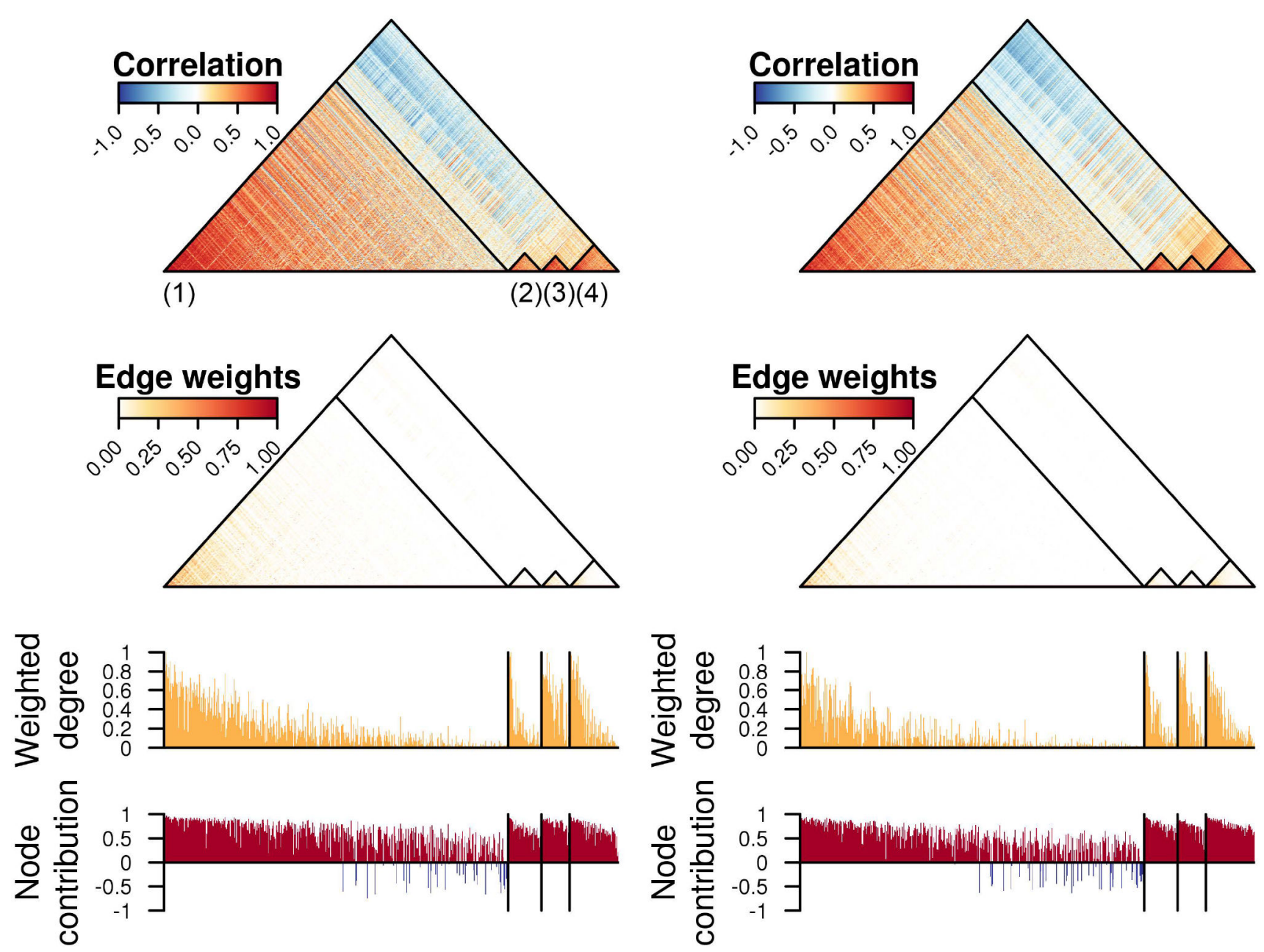

Figure 4: Preservation of obese asthma modules in CHS and GACRS.

The first (top) panel shows a heatmap of pair-wise correlations among the genes comprising the black (1), lightcyan (2), grey60 (3), and midnightblue (4) modules [see 'cor.cor' and 'avg.cor' metrics defined in Table S6]. The second panel shows a heatmap of the edge weights (connections) among the genes comprising the four modules ['avg.weight']. The third panel shows the distribution of scaled weight degrees (relative connectedness) among the genes comprising the four modules ['cor.degree']. The fourth panel shows the distribution of node contributions (correlation to module eigengene) among the genes comprising the four modules ['cor.contrib' and 'avg.contrib']. The 'coherence' metric is not summarized in this figure. Genes are ordered from left to right based on their weighted degree in CAMP (the discovery cohort) to highlight the consistency of the network properties in CHS and GACRS (the replication cohorts). 
Table 1:

\section{CAMP discovery cohort characteristics.}

To test for differences between asthmatic subjects with and without adult obesity in the CAMP cohort, Welch two sample $t$-tests were used for continuous traits, and chi-squared tests were used for proportions. Medication use is reported for the seven days preceding the assaying of gene expression. ICS, inhaled corticosteroids; LTRA, leukotriene receptor antagonists; LABA, long-acting beta agonists.

\begin{tabular}{|c|c|c|c|}
\hline$N$ & $\begin{array}{c}\text { Obese } \\
104\end{array}$ & $\begin{array}{c}\text { Non-Obese } \\
410\end{array}$ & $\begin{array}{c}\text { Difference } \boldsymbol{P} \\
-\end{array}$ \\
\hline Childhood BMI percentile & $77.8(15.5)$ & $53.1(26.3)$ & $4.18 \mathrm{E}-28$ \\
\hline Adult BMI & $31.1(3.9)$ & $23.5(3.3)$ & $4.30 \mathrm{E}-39$ \\
\hline Age at asthma onset (S.D.) [yrs.] & $3.6(2.6)$ & $3.9(2.4)$ & 0.27 \\
\hline Age at study enrollment (S.D.) [yrs.] & $9.1(2.0)$ & $8.7(2.1)$ & 0.09 \\
\hline Age at gene expression (S.D.) [yrs.] & $21.2(2.1)$ & $20.8(2.2)$ & 0.15 \\
\hline$\%$ Female & 37.5 & 38.0 & 1.00 \\
\hline \multicolumn{4}{|l|}{ Race/Ethnicity $N(\%)$} \\
\hline European & $67(64.4)$ & $294(71.7)$ & \multirow{4}{*}{0.02} \\
\hline Black/African American & $18(17.3)$ & $53(12.9)$ & \\
\hline Hispanic/Latino & $16(15.4)$ & $31(7.6)$ & \\
\hline Other & $3(2.9)$ & $32(7.8)$ & \\
\hline $\log _{10} \operatorname{IgE}$ & $2.5(0.6)$ & $2.6(0.6)$ & 0.28 \\
\hline \% Eosinophils (S.D.) & $3.8(2.7)$ & $4.1(2.9)$ & 0.29 \\
\hline \% Lymphocytes (S.D.) & $30.8(7.5)$ & $31.6(8.1)$ & 0.40 \\
\hline \% Monocytes (S.D.) & $7.5(2.3)$ & $7.6(2.2)$ & 0.54 \\
\hline \% Neutrophils (S.D.) & $57.5(8.8)$ & $56.0(9.4)$ & 0.18 \\
\hline$\%$ ICS use & 26.5 & 22.7 & 1.00 \\
\hline \% LTRA use & 5.9 & 5.6 & 1.00 \\
\hline \% LABA use & 17.6 & 12.1 & 0.19 \\
\hline \% Any asthma medication use & 27.5 & 20.0 & 0.71 \\
\hline
\end{tabular}




\section{Table 2:}

\section{Epidemiological eigengene-phenotype model of obese asthma in CAMP.}

Features are sorted by significance in the adjusted eigengene model. Nominally significant features $(P<0.05)$ are marked in bold. Race and sex were treated as factors, with reference levels of "European" and "Female", respectively. The race variable from Table 1 was also collapsed into "European" and "Non-European" (70\% and $30 \%$, respectively), because of small group sizes for "Black/African American", "Hispanic/Latino", and "Other". A subset of 112 subjects was missing measured blood count data, but there was no significant difference in expression of any of the four eigengenes between subjects with and without those data (all $P>$ 0.1). Medication use is reported for the seven days preceding the assaying of gene expression. ME, module eigengene; ICS, inhaled corticosteroids; LTRA, leukotriene receptor antagonists; LABA, long-acting beta agonists.

\begin{tabular}{lcccccc}
\hline & \multicolumn{3}{c}{ Unadjusted Eigengene Model } & \multicolumn{3}{c}{ Adjusted Eigengene Model } \\
\multicolumn{1}{c}{ Parameter } & Beta & SE & $\mathbf{P}$ & Beta & SE & P \\
\hline Modules & & & & & & \\
MEblack & 12.65 & 3.36 & $\mathbf{1 . 6 7 E - 0 4}$ & 15.19 & 4.11 & $\mathbf{2 . 2 0 E - 0 4}$ \\
MEmidnightblue & 9.17 & 2.98 & $\mathbf{2 . 1 0 E - 0 3}$ & 9.12 & 3.76 & $\mathbf{1 . 5 4 E - 0 2}$ \\
MEgrey60 & 7.83 & 2.97 & $\mathbf{8 . 3 2 E - 0 3}$ & 8.68 & 3.66 & $\mathbf{1 . 7 7 E - 0 2}$ \\
MElightcyan & -7.58 & 2.85 & $\mathbf{7 . 8 2 E - 0 3}$ & -5.09 & 6.42 & $4.27 \mathrm{E}-01$ \\
Covariates (adjusted model only) & & & & & \\
Childhood BMI percentile & & & 0.05 & 0.01 & $\mathbf{1 . 4 9 E - 1 0}$ \\
Age at expression & & & & 0.09 & 0.07 & $1.96 \mathrm{E}-01$ \\
Sex & & & & 0.27 & 0.30 & $3.81 \mathrm{E}-01$ \\
\% Monocytes & & & & 0.07 & 0.13 & $6.13 \mathrm{E}-01$ \\
ICS use & & & 0.24 & 0.49 & $6.30 \mathrm{E}-01$ \\
\% Neutrophils & & & & 0.06 & 0.12 & $6.44 \mathrm{E}-01$ \\
\% Eosinophils & & & & 0.06 & 0.15 & $6.57 \mathrm{E}-01$ \\
\% Lymphocytes & & & & 0.04 & 0.13 & $7.29 \mathrm{E}-01$ \\
LABA use & & & & 0.07 & 0.59 & $9.07 \mathrm{E}-01$ \\
LTRA use & & & & 0.07 & 0.63 & $9.08 \mathrm{E}-01$ \\
Race & & & & & & \\
\hline
\end{tabular}




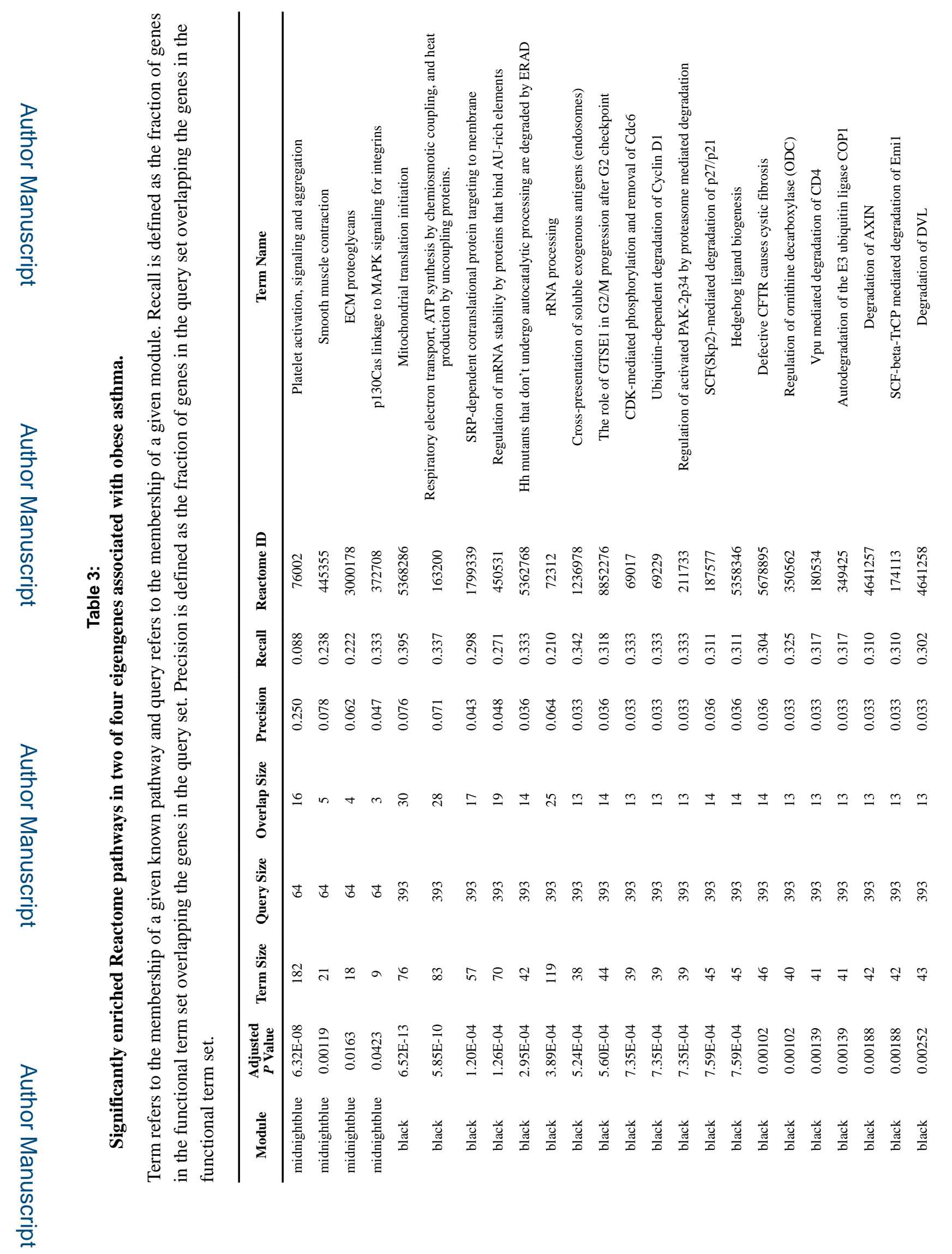

Obesity (Silver Spring). Author manuscript; available in PMC 2019 December 01. 


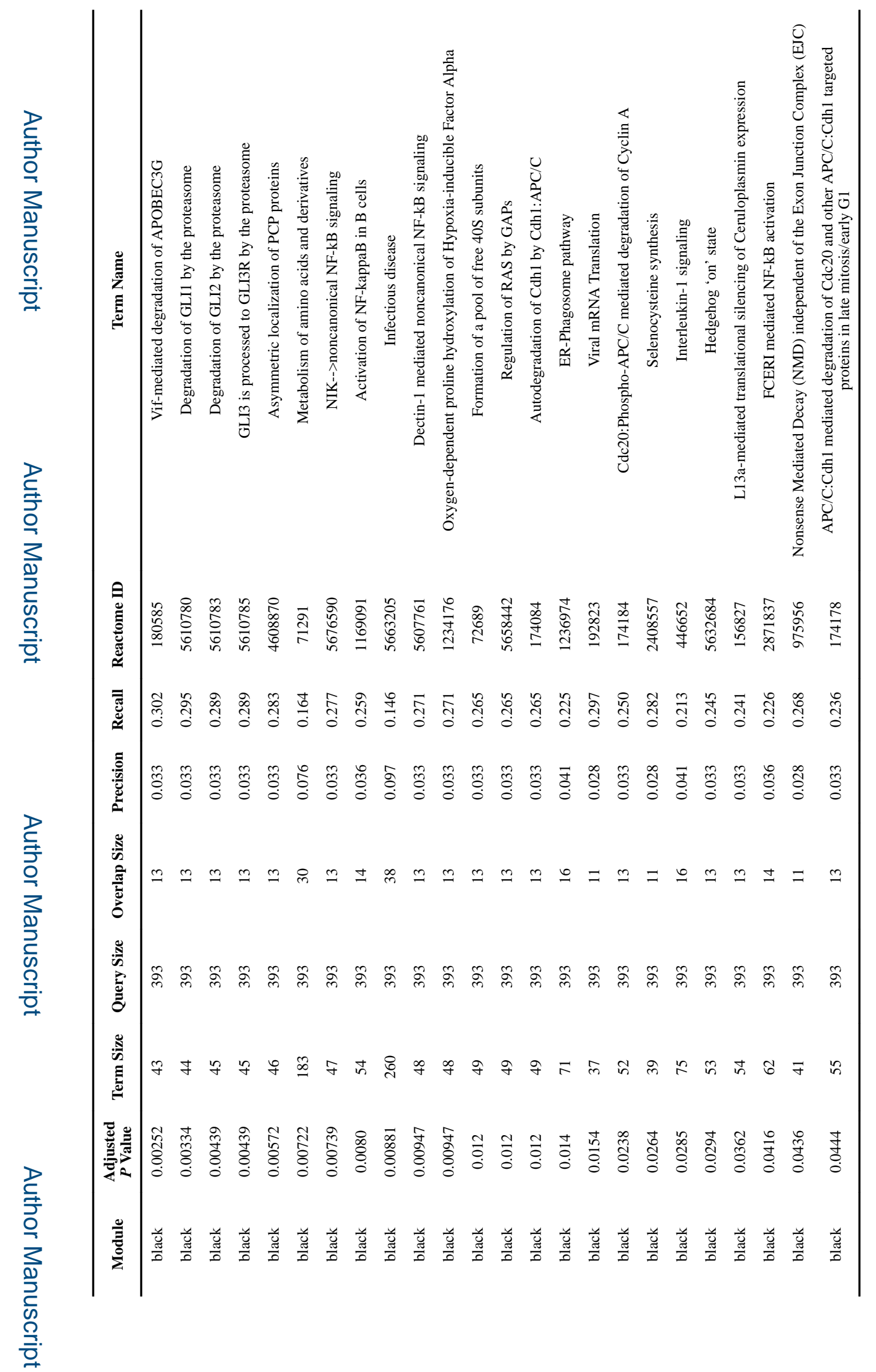

Obesity (Silver Spring). Author manuscript; available in PMC 2019 December 01. 
Table 4:

Enrichments for differential expression of obese asthma module gene sets.

Significant enrichments $(P<0.05)$ with a consistent direction of effect to results from Table 2 are marked in bold. ES, enrichment score. Two random subsets of the null module were used as negative control sets.

\begin{tabular}{|c|c|c|c|c|c|c|c|}
\hline \multirow[b]{3}{*}{ Gene Set } & \multirow[b]{3}{*}{ Set Size } & \multirow{2}{*}{\multicolumn{2}{|c|}{$\begin{array}{c}\text { Discovery } \\
\text { CAMP }(n=514)\end{array}$}} & \multicolumn{4}{|c|}{ Replication } \\
\hline & & & & \multicolumn{2}{|c|}{ CHS $(n=91)$} & \multicolumn{2}{|c|}{ GACRS $(n=329)$} \\
\hline & & ES & $P$ & ES & $P$ & ES & $P$ \\
\hline black & 464 & 0.058 & $5.0 \mathrm{E}-58$ & 0.001 & $8.9 \mathrm{E}-01$ & 0.035 & 3.3E-33 \\
\hline grey60 & 38 & 0.030 & $1.5 \mathrm{E}-11$ & 0.051 & 5.4E-07 & 0.020 & 7.5E-06 \\
\hline lightcyan & 45 & -0.005 & $9.8 \mathrm{E}-02$ & -0.049 & 5.4E-07 & -0.038 & 3.8E-07 \\
\hline midnightblue & 66 & 0.063 & $1.2 \mathrm{E}-11$ & 0.038 & 4.6E-06 & 0.029 & 9.6E-07 \\
\hline \multicolumn{8}{|c|}{ Negative controls } \\
\hline null & 200 & 0.000 & $5.0 \mathrm{E}-01$ & -0.008 & $9.6 \mathrm{E}-02$ & -0.006 & $5.3 \mathrm{E}-03$ \\
\hline null & 50 & 0.001 & $8.4 \mathrm{E}-01$ & 0.007 & $7.2 \mathrm{E}-01$ & -0.011 & $6.1 \mathrm{E}-02$ \\
\hline
\end{tabular}

